\title{
Measurement of Three-Dimensional Dipole Orientation of a Single Fluorescent Nanoemitter by Emission Polarization Analysis
}

\author{
Clotilde Lethiec, ${ }^{1,2}$ Julien Laverdant, ${ }^{1,2,3}$ Henri Vallon, ${ }^{1,2}$ Clémentine Javaux, ${ }^{4}$ Benoît Dubertret, ${ }^{4}$ \\ Jean-Marc Frigerio, ${ }^{1,2}$ Catherine Schwob, ${ }^{1,2}$ Laurent Coolen, ${ }^{1,2}$ and Agnès Maître ${ }^{1,2, *}$ \\ ${ }^{1}$ Sorbonne Universités, UPMC Univ. Paris 06, , UMR 7588, Institut des NanoSciences de Paris, \\ F-75005 Paris, France \\ ${ }^{2}$ CNRS, UMR 7588, Institut des NanoSciences de Paris, F-75005 Paris, France \\ ${ }^{3}$ ILM, Université de Lyon, Université Lyon-1, UMR CNRS 5306, 69622 Villeurbanne, France \\ ${ }^{4}$ LPEM, ESPCI/CNRS/UPMC UMR 8213, 10, Rue Vauquelin, 75005 Paris, France \\ (Received 4 August 2013; revised manuscript received 19 November 2013; published 28 May 2014)
}

\begin{abstract}
We demonstrate theoretically and experimentally that the three-dimensional orientation of a single fluorescent nanoemitter can be determined by polarization analysis of the emitted light (while excitation polarization analysis provides only the in-plane orientation). The determination of the emitter orientation by polarimetry requires a theoretical description, including the objective numerical aperture, the $1 \mathrm{D}$ or $2 \mathrm{D}$ nature of the emitting dipole, and the environment close to the dipole. We develop a model covering most experimentally relevant microscopy configurations and provide analytical relations that are useful for orientation measurements. We perform polarimetric measurements on high-quality core-shell $\mathrm{CdSe} / \mathrm{CdS}$ nanocrystals and demonstrate that they can be approximated by two orthogonal degenerated dipoles. Finally, we show that the orientation of a dipole can be inferred by polarimetric measurement, even for a dipole in the vicinity of a gold film, while in this case, the well-established defocused microscopy is not appropriate.
\end{abstract}

DOI: 10.1103/PhysRevX.4.021037

\section{INTRODUCTION}

The determination of the orientation of a single photoluminescent emitter has been a major issue since early single-molecule studies [1-5]. It is a valuable tool for understanding distortion mechanisms in polymers [6] or biological systems $[7,8]$. For nano-optics and plasmonics, the orientation of an emitter has a strong influence on its coupling to the environment [9-13]. Over the last few years, large efforts have been made in order to deterministically couple emitters to photonic nanostructures. Spatial positioning of an emitter [14,15] and spectral tuning of its emission to cavity modes [16] by deterministic methods have already been successfully implemented by several groups. However, up to now the deterministic control of the orientation of a dipole inside a nanostructure still remains a challenge, although it is crucial for efficient coupling. In this paper, we develop a practical method to determine the orientation of a single dipole, which is a first step for deterministic coupling to nanostructures. Early orientation studies relied on polarized excitation [17]. The electric field

\footnotetext{
*agnes.maitre@insp.upmc.fr
}

Published by the American Physical Society under the terms of the Creative Commons Attribution 3.0 License. Further distribution of this work must maintain attribution to the author(s) and the published article's title, journal citation, and DOI.
Subject Areas: Nanophysics, Optics, Photonics

at the focal point thus mainly probes the in-plane component of the dipole, and its azimuthal orientation $\Phi$ can be inferred, but the (out-of-plane) polar orientation $\Theta$ remains unknown [18]. In order to measure $\Theta$, various sophisticated schemes have been proposed to increase the out-of-plane component of the electric field [4,19-23].

Moreover, most of these works intrinsically probe the orientation of the absorbing dipole, which for nonresonant photoluminescence can be extremely different from the emitting dipole. Depending on their group symmetry, molecules can have orthogonal excited and emitting dipoles [24]. As for colloidal semiconductor nanocrystals, while they show little dependence on the excitation polarization [25], because of their dense continuum of absorption levels, their emission is usually described as a sum of two incoherent orthogonal dipoles [26-28]. The orientation of such a nanocrystal can thus only be obtained from its emission properties, as its excitation properties are isotropic.

Various methods have been suggested to determine the orientation $\Theta$ of emitting dipoles. A successful method has been defocused imaging [8,9,29-34], which offers the most convenient implementation. Its precision requires the defocusing of emitting pattern on many pixels of a camera. Therefore, it consumes photons and requires bright emitters and highly sensitive detectors. Moreover, it can only be applied for configurations for which the emission diagram is highly dependent on dipole orientation. Recently, several authors have extended this method to super-resolution 
experiments $[35,36]$. In order to overcome these limitations, some authors propose different variants. They mostly rely (except for Ref. [10], which uses relative lifetime differences) on taking advantage of standard [22] or tailored aberrations [37]. Another ingenuous method, developed in Ref. [38], is based on probing the emission diagram by decomposing the different emission directions with an annular separating plate. This efficient technique does not require a large number of photons but suffers from an eightfold degeneracy, preventing the exact determination of the orientation. Besides, this measurement is highly sensitive to the positioning on the annular plate. It is possible that, by analogy with the idea that the out-of-plane component of the excitation dipole cannot be probed by polarization analysis, for the emission dipole, only a few studies have considered polarization analysis for orientation measurements [26]. The pioneering theoretical model developed in Ref. [39] proposes determining the orientation of a dipole inside a homogeneous infinite dielectric by polarimetric analysis. A first experimental implementation has been described in Ref. [40]. It relies on only four measurements, which makes this method very sensitive to any intensity fluctuations. In this paper, we show that the three-dimensional orientation $(\Theta, \Phi)$ of a nanoemitter can be obtained by analyzing its emission polarization on avalanche photodiodes, even in the case of a fluctuating emission. We insist on the fact that the emitter optical environment (such as proximity to an interface) must be taken into account. We provide an analytical model that can be used to interpret the data and extract $(\Theta, \Phi)$ in a wide range of realistic experimental conditions. We demonstrate this experimentally by measuring the orientation of highquality thick-shell CdSe/CdS nanocrystals with 2D-dipolar emission, including the case when the emitter lies in proximity to a gold film, a situation for which the more standard defocused imaging is not sufficiently sensitive to provide reliable information.

In Sec. II, we present the elements of our theoretical model. In Sec. III, we propose a method to determine the 1D or 2D nature of dipoles and show experimentally that our $\mathrm{CdSe} / \mathrm{CdS}$ nanocrystals are 2D dipoles. In Sec. IV, we develop the results of the theoretical model and show that the orientation can be extracted from polarization data. We implement this experimentally in Sec. V and measure the orientation of various nanocrystals. In Sec. VI, we consider the case of a nanocrystal near a metallic film and show that the orientation can be obtained from polarization analysis but not from defocused imaging.

\section{THEORETICAL FRAMEWORK}

In this section we develop the theoretical modeling used in this study. The simulated situation is illustrated in Figs. 1(a) and 1(b): The emission of a dipole is collected by an objective and analyzed by a rotating polarizer. The polarizer orientation angle $\alpha$ is continuously rotated. The

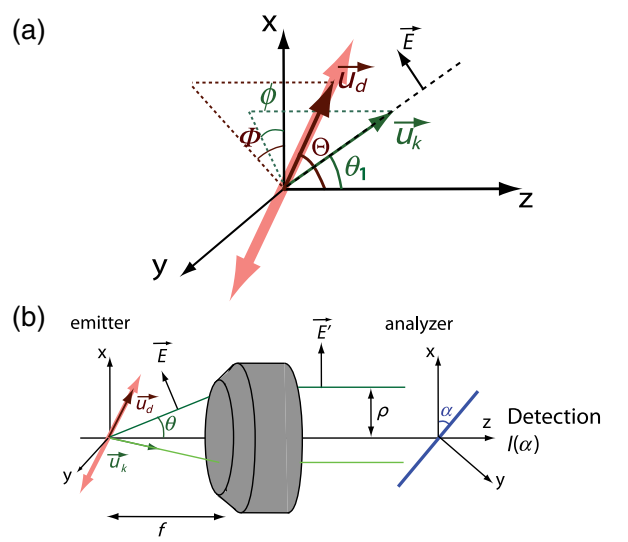

FIG. 1. (a) Schematic of a normalized dipole $\vec{u}_{d}$ with in-plane angle $\Phi$ and off-axis angle $\Theta$ and a normalized wave vector $\vec{u}_{k}$ with in-plane angle $\phi$ and off-axis angle $\theta_{1}$. (b) Schematic of the simulated situation: the dipole described above, a microscope objective, and a polarizer with in-plane angle $\alpha$.

principle of the measurement is to extract, from the detected intensity $I(\alpha)$, the azimuthal angle $\Phi$ and the polar angle $\Theta$ of the emitting dipole.

The far-field emission component of the electric field emitted by a linear dipole (1D dipole) into a direction $\left(\theta_{1}, \phi\right)$ can be expressed as

$$
\vec{E}\left(\theta_{1}, \phi\right)=\frac{D}{r}\left(\vec{u}_{k} \wedge \vec{u}_{d} \wedge \vec{u}_{k}\right),
$$

with $D$ a constant depending on the refractive index of the medium containing the dipole, where

$$
\begin{gathered}
\vec{u}_{d}=\left(\begin{array}{c}
\sin \Theta \cos \Phi \\
\sin \Theta \sin \Phi \\
\cos \Theta
\end{array}\right), \\
\vec{u}_{k}=\frac{1}{k_{1}}\left(\begin{array}{c}
k_{1_{\|}} \cos \phi \\
k_{1_{\|}} \sin \phi \\
k_{1_{z}}
\end{array}\right)=\left(\begin{array}{c}
\sin \theta_{1} \cos \phi \\
\sin \theta_{1} \sin \phi \\
\cos \theta_{1}
\end{array}\right)
\end{gathered}
$$

are the unit vectors corresponding to the dipole orientation and to the considered emission $\vec{k}_{1}$-vector direction, respectively.

Equation (1) expresses the emission of a dipole in a homogeneous dielectric environment, which can be decomposed into its $s$ and $p$ components

$$
\vec{E}\left(\theta_{1}, \phi\right)=\frac{D}{r}\left(E_{s}\left(\theta_{1}, \phi\right) \vec{u}_{s}+E_{p}\left(\theta_{1}, \phi\right) \vec{u}_{p}\right),
$$

with the unit vectors

$$
\vec{u}_{s}=\left(\begin{array}{c}
\sin \phi \\
-\cos \phi \\
0
\end{array}\right) \quad \text { and } \quad \vec{u}_{p}=\left(\begin{array}{c}
\cos \theta_{1} \cos \phi \\
\cos \theta_{1} \sin \phi \\
-\sin \theta_{1}
\end{array}\right)
$$


and

$$
\begin{gathered}
E_{s}\left(\theta_{1}, \phi\right)=\sin \Theta \sin (\phi-\Phi), \\
E_{p}\left(\theta_{1}, \phi\right)=\left(E_{p_{a}}\left(\theta_{1}, \phi\right)+E_{p_{b}}\left(\theta_{1}, \phi\right)\right),
\end{gathered}
$$

with

$$
\begin{gathered}
E_{p_{a}}\left(\theta_{1}, \phi\right)=-\cos \Theta \sin \theta_{1}, \\
E_{p_{b}}\left(\theta_{1}, \phi\right)=\sin \Theta \cos \theta_{1} \cos (\Phi-\phi) .
\end{gathered}
$$

However, in many experimental observation conditions, the dipole is in the vicinity of an optical interface, which modifies the emission diagram and polarization [41]. We describe here five relevant experimental conditions, as presented in Fig. 2. Aside from the case of a homogeneous medium (i), we consider the following: (ii) a sample with emitters deposited on a planar substrate of index $n_{1}$ and protected by a polymer layer of thickness $d$ of the same index, observed with an immersion objective, the upper medium (most likely air) being of index $n_{2}$, (iii) a sample with emitters at a distance $\mathrm{d}$ (with $\mathrm{d}$ tending towards 0 ) from a planar surface (substrate index $n_{2}$ ) without any protecting layer, observed with an air objective (air index $n_{1}$ ), (iv) a sample with emitters on a planar surface with a polymer protecting layer (index $n_{1}$ ), observed with an air objective (air index $n_{2}$ ), (v) a sample with emitters at a distance $\mathrm{d}$ (with $\mathrm{d}$ tending towards 0 ) from a planar surface (index $n_{1}$ ) without any protecting layer, observed with an immersion objective (index $n_{2}$ ).

For all situations, we use $n_{1}$ for the index of the medium containing the emitter [a glass index for (i), (ii), (iv) and an air index for (iii) and (v)] and $n_{2}$ for the index of the other medium [a glass index for (iii) and (v) and an air index for (ii) and (iv)].
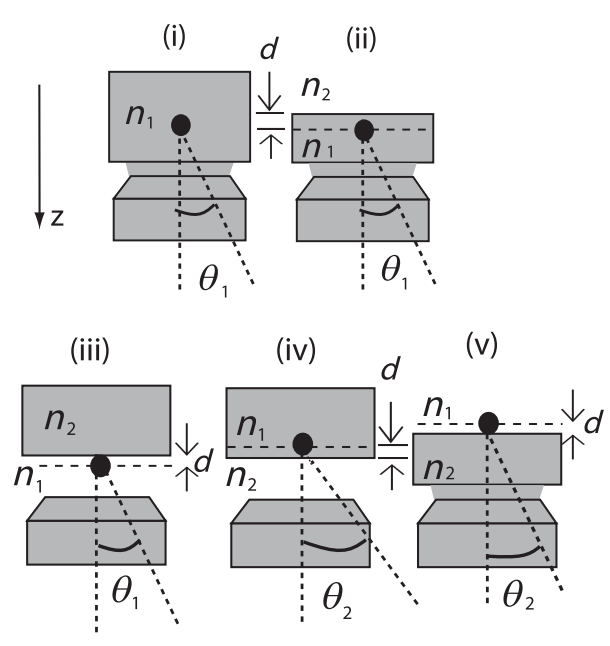

FIG. 2. The five cases corresponding to different experimental conditions, numbered from (i) to (v).
For situations (ii) and (iii), the detected electric field is a sum of the direct emitted field and its reflected fields, whereas in situations (iv) and (v), only the direct emission is collected, after transmission through the interface. In the last situation (v), we need to take into account, for the detected field, the evanescent component of the dipole's near-field emission, which becomes propagative when transmitted through the interface [42]. This particular case is developed in the Appendix.

The presence of a near interface can be described generally by multiplying $E_{s}, E_{p_{a}}$, and $E_{p_{b}}$ by the functions $f_{s}, f_{p_{a}}$, and $f_{p_{b}}$, whose definition depends on whether it involves reflection or transmission of the emitted field [41].

\begin{tabular}{lccc}
\hline & $f_{s}=$ & $f_{p_{a}}=$ & $f_{p_{b}}=$ \\
\hline (i) & 1 & 1 & 1 \\
(ii) & $1+r_{s}^{12} e^{i \Delta}$ & $1+r_{p}^{12} e^{i \Delta}$ & $1-r_{p}^{12} e^{i \Delta}$ \\
(iii) & $1+r_{s}^{12} e^{i \Delta}$ & $1+r_{p}^{12} e^{i \Delta}$ & $1-r_{p}^{12} e^{i \Delta}$ \\
(iv) & $t_{s}^{12}$ & $t_{p}^{12}$ & $t_{p}^{12}$ \\
\hline
\end{tabular}

Here, $\Delta\left(\theta_{1}\right)=4 \pi n_{1} d \cos \left(\theta_{1}\right) / \lambda$, and the Fresnel reflection and transmission coefficients are

$$
\begin{aligned}
r_{s}^{12} & =\frac{n_{1} \cos \theta_{1}-n_{2} \cos \theta_{2}}{n_{1} \cos \theta_{1}+n_{2} \cos \theta_{2}}, \\
r_{p}^{12} & =\frac{n_{2} \cos \theta_{1}-n_{1} \cos \theta_{2}}{n_{1} \cos \theta_{2}+n_{2} \cos \theta_{1}}, \\
t_{s}^{12} & =\frac{2 n_{1} \cos \theta_{1}}{n_{1} \cos \theta_{1}+n_{2} \cos \theta_{2}}, \\
t_{p}^{12} & =\frac{2 n_{1} \cos \theta_{1}}{n_{1} \cos \theta_{2}+n_{2} \cos \theta_{1}},
\end{aligned}
$$

with

$$
n_{1} \sin \theta_{1}=n_{2} \sin \theta_{2} .
$$

The field emitted by the pointlike dipole collected by the objective is collimated after the lens. After passing through the objective of focal length $f$ (neglecting aberrations) and taking into account the apodization factor $\left(\frac{\cos \theta}{n_{j}}\right)^{-\frac{1}{2}}$ [42], where $j=1$ in cases (ii) and (iii) and $j=2$ in cases (iv) and (v), this field becomes

$$
\vec{E}^{\prime}\left(\theta_{j}, \phi\right)=\frac{1}{f}\left(\frac{\cos \theta}{n_{j}}\right)^{-\frac{1}{2}} \overrightarrow{\mathcal{E}},
$$

where

$$
\begin{aligned}
\overrightarrow{\mathcal{E}}\left(\theta_{1}, \phi\right)= & D \frac{n_{j}}{n_{1}}\left(E_{s}\left(\theta_{1}, \phi\right) f_{s}\left(\theta_{1}\right) \vec{v}_{s}\right. \\
& \left.+\left[E_{p_{a}}\left(\theta_{1}, \phi\right) f_{p_{a}}\left(\theta_{1}\right)+E_{p_{b}}\left(\theta_{1}, \phi\right) f_{p_{b}}\left(\theta_{1}\right)\right] \vec{v}_{p}\right),
\end{aligned}
$$


with the new unit vectors

$$
\vec{v}_{s}=\vec{u}_{s} \quad \text { and } \quad \vec{v}_{p}=\left(\begin{array}{c}
\cos \phi \\
\sin \phi \\
0
\end{array}\right) .
$$

The objective collects the light for all values of $\phi$ between 0 and $2 \pi$ and for $\theta_{j}$ between 0 and $\theta_{j \max }$. The maximum collection angle $\theta_{j \max }$ is related to the objective numerical aperture $N A$ by $\theta_{j \max }=\mathrm{a} \sin \left(N A / n_{j}\right)$.

Finally, a polarizer is set after the lens, along a unit vector $\vec{u}_{\alpha}$ at an angle $\alpha$ from the $x$ axis, so that the normalized emitted power detected after the polarizer is

$$
P(\alpha)=\int_{\rho=0}^{\rho_{\max }} \int_{\phi=0}^{2 \pi}\left|\vec{E}^{\prime}\left(\theta_{j}, \phi\right) \cdot \vec{u}_{\alpha}\right|^{2} \rho d \rho d \phi,
$$

with the sine condition [43]

$$
\rho=f \sin \theta_{j},
$$

which leads to

$$
P(\alpha)=\int_{\theta_{j}=0}^{\theta_{j \max }} \int_{\phi=0}^{2 \pi} f^{2}\left|\vec{E}^{\prime}\left(\theta_{j}, \phi\right) \cdot \vec{u}_{\alpha}\right|^{2} \cos \theta_{j} \sin \theta_{j} d \theta_{j} d \phi .
$$

For the transmission case (iv), the conservation of power per solid angle at the interface is assured by taking into account the apodization factor $\left(\cos \theta_{2} / \cos \theta_{1}\right)^{2}$.

Finally, for cases (i)-(iv), the emitted power is expressed as

$$
\begin{aligned}
P(\alpha)= & \int_{\varphi=0}^{2 \pi} \int_{\theta_{1}=0}^{\theta_{1 \max }}\left|\overrightarrow{\mathcal{E}}\left(\theta_{1}, \varphi\right) \cdot \vec{u}(\alpha)\right|^{2} n_{j}\left(\frac{n_{1}}{n_{2}}\right)^{2} \\
& \times \frac{\cos \theta_{2}}{\cos \theta_{1}} \sin \theta_{1} d \theta_{1} d \varphi .
\end{aligned}
$$

For the particular case (v), the calculations are detailed in the Appendix.

\section{DETERMINATION OF THE EMITTING DIPOLE DIMENSION}

Up to now, we have considered the case of the textbook linear dipole, which will hereafter be called the "1D dipole." However, in many cases, the emission originates from a twofold degenerated emitting energy level. It can be written as an incoherent sum of two linear degenerated orthogonal dipoles and is referred to as the "2D dipole." The excitation at energies well above the energy of emission is followed by a thermalization on two degenerated emitting levels, so it induces the emission on two incoherent transitions. Such a situation has been reported for some molecules such as benzene [24], for some nitrogen-vacancy centers [44], and for $\mathrm{CdSe} / \mathrm{ZnS}$ colloidal nanocrystals at low [26] and room temperatures [27,28]. The latter observation is related to theoretical calculations of the emitting state's fine structure [45], predicting that the lowest allowed transition is twofold degenerate. Accordingly, a "3D dipole" would be an isotropic emitter, corresponding to the incoherent sum of three linear dipoles. The issue of dimensionality is then a general question for all emitters and has to be determined to retrieve the orientation of a dipole.

The 3D orientation of a 1D dipole corresponds to the orientation of the dipole itself, whereas for a $2 \mathrm{D}$ dipole it corresponds to the orientation of the axis perpendicular to the plane that contains the two dipoles.

In this section, we characterize, experimentally, the 1D or $2 \mathrm{D}$ nature of the emitters considered in this paper. In order to measure the dimension of a dipole, we use the experimental setup suggested by Chung et al. [27] [Fig. 3(a)], which shows simultaneously the $\mathrm{x}$ - and $y$-polarized emission of the same emitters on two charge-coupled-device (CCD) cameras situated after a polarizing beam splitter. For practical reasons, the polarizing beam splitter cube in our experiment had to be placed not just after the objective lens but between the microscope and the CCD detector. However, we can consider that the optical beam is quasicollimated in the first approximation because of the high value of the lens magnification (x100), so our theory remains valid. The sample is prepared in configuration (ii): a glass sample covered by $d=50 \mathrm{~nm}$ of polymer of index $n_{1}=1.5$, with air index $n_{2}=1$ and a numerical aperture equal to 1.4 .
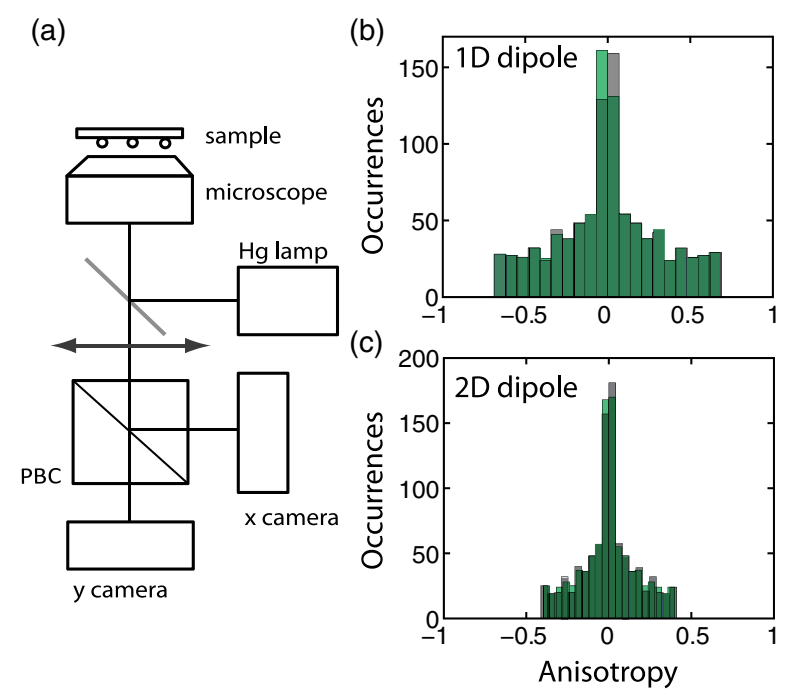

FIG. 3. (a) Schematic of the setup. A polarizing beam-splitter cube (PBC) is placed in front of two CCD cameras (directions $\mathrm{x}$ and $\mathrm{y})$. (b) and (c) Distribution of the anisotropy $\mathcal{A}=\left(I_{x}-\right.$ $\left.I_{y}\right) /\left(I_{x}+I_{y}\right)$ simulated for randomly oriented $1 \mathrm{D}$ dipoles and 2D dipoles. Calculations are performed for situation (ii), with $n_{1}=1.5, n_{2}=1, d=50 \mathrm{~nm}, \lambda=620$ (grey) and $565 \mathrm{~nm}$ (green) and a 1.4 numerical aperture. 
It is then possible to show many emitters and for each emitter to measure the intensities $I_{x}$ and $I_{y}$ on the two cameras and define a polarization anisotropy $\mathcal{A}$ :

$$
\mathcal{A}=\frac{I_{x}-I_{y}}{I_{x}+I_{y}} .
$$

On the other hand, $\mathcal{A}$ can be calculated, for a given dipole with an orientation $(\Theta, \Phi)$, from Eq. (20) with

$$
\begin{gathered}
I_{x}=I(\alpha=0), \\
I_{y}=I\left(\alpha=\frac{\pi}{2}\right) .
\end{gathered}
$$

We assume a collection of emitters with random orientations isotropically distributed and plot a histogram of the calculated anisotropies, for 1D and 2D dipoles [see Fig. 3(b); we plot the two wavelengths 565 and $620 \mathrm{~nm}$, as they will be relevant for the experiment below, although the results are very close]. Both histograms display a peak at $\mathcal{A}=0$, but the extension of the wings on either side of this peak is different: For a 1D dipole, the wings extend up to \pm 0.7 , while for a $2 \mathrm{D}$ dipole, they extend only to \pm 0.4 . It is thus possible, by measuring the anisotropy of randomly oriented emitters and plotting a histogram of these anisotropies, to discriminate whether this type of emitter is $1 \mathrm{D}$ or 2D. Let us note that this calculation is performed for situation (ii) with the parameters indicated above: In a different configuration, the results would be quantitatively slightly different, but the qualitative difference between 1D and 2D dipoles would remain.

The experimental results obtained for different types of emitters are shown in Fig. 4.

Latex beads infiltrated with dye molecules (Lifetechnologies, F8763, emission peak at $600 \mathrm{~nm}$ ) are first studied in order to validate the method. Each bead contains a large number of emitters so that they can be considered as pointlike isotropic emitters: The polarization anisotropy should be zero for all beads. Indeed, we obtain [Fig. 4(a), error bar: 0.05] a peak centered on $\mathcal{A}=0$, with a width of 0.05 attributed to measurement uncertainties.

We then study colloidal core/shell $\mathrm{CdSe} / \mathrm{CdS}$ nanocrystals (emission $620 \mathrm{~nm}$, core diameter $2.5 \mathrm{~nm}$, total diameter $13 \mathrm{~nm}$ ) exhibiting very good brightness and suppressed blinking [46] [Fig. 4(d)]. The obtained anisotropy histogram [Fig. 4(b), error bar: 0.05] presents a peak on zero, and the extension of the curve reaches \pm 0.4 . This experimental curve is in good agreement with the theoretical curve of Fig. 3(c), taking into account a slight broadening of the central peak due to the 0.05 uncertainty on $\mathcal{A}$. This demonstrates that these nanocrystals are 2D dipoles. If they were $1 \mathrm{D}$ dipoles, their anisotropy histogram would extend up to \pm 0.7 .

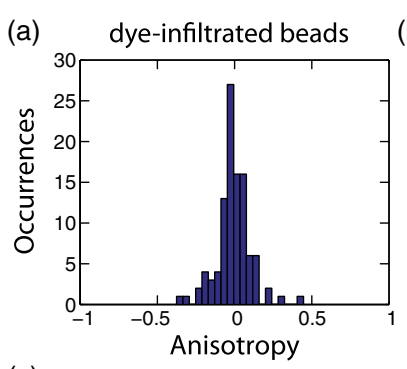

(c) CdSe/ZnS nanocrystals
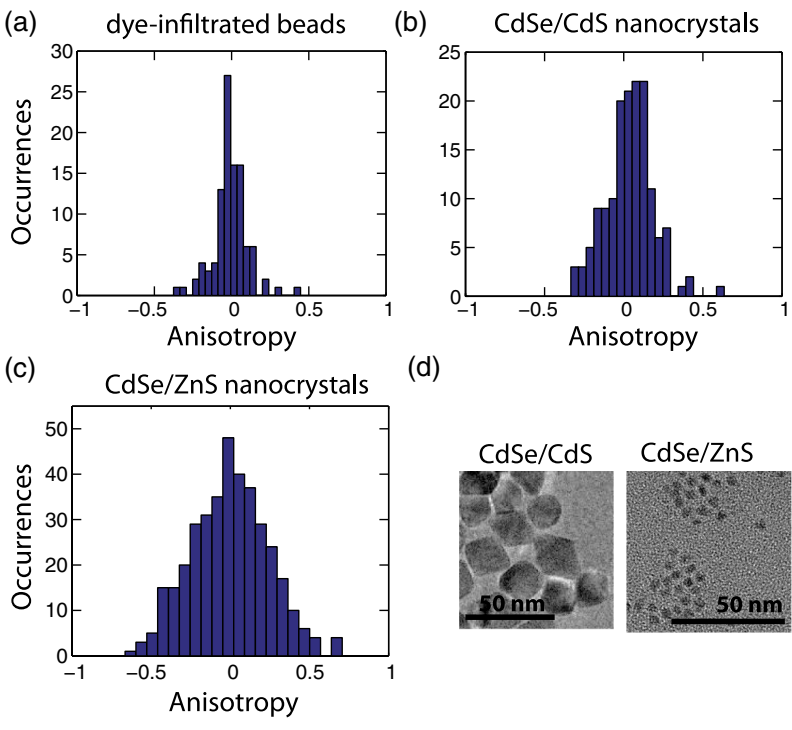

(d)

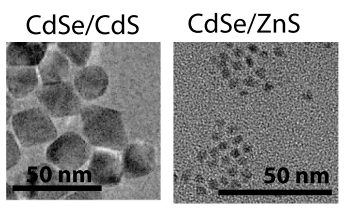

FIG. 4. Distribution of the anisotropy $\mathcal{A}=\left(I_{x}-I_{y}\right) /\left(I_{x}+I_{y}\right)$ measured for 103 beads (a), $152 \mathrm{CdSe} / \mathrm{CdS}$ nanocrystals (b), and $374 \mathrm{CdSe} / \mathrm{ZnS}$ nanocrystals (c). Experimental conditions: situation (ii), numerical aperture $1.4, d=50 \mathrm{~nm}$ of PMMA of index 1.5. (d) Transmission electron microscopy image of the CdSe/ $\mathrm{CdS}$ and $\mathrm{CdSe} / \mathrm{ZnS}$ nanocrystal samples.

Finally, we consider core/shell CdSe/ZnS nanocrystals (QDots, Invitrogen, emission $565 \mathrm{~nm}$ ) [Fig. 4(d)]. For such nanocrystals, Empedocles et al. have reported a 2D-dipole behavior [26]. The experimental histogram obtained for these nanocrystals is plotted in Fig. 4(c). The error bar is more important here (0.1) because the emission intensity is lower for these emitters. The curve presents a peak at zero and wings extending to \pm 0.5 . The agreement with the theoretical histogram for $2 \mathrm{D}$ dipoles is not very good, possibly because of a mixture of $1 \mathrm{D}$ and $2 \mathrm{D}$ dipolar emission as suggested in Refs. [47] and [48]. This can be explained by an energy splitting, at room temperature, smaller than $k_{B} T$ between the degenerated $\pm 1^{L}$ and the linear $0^{L}$ transitions, which allows a linearly polarized emission [45].

\section{THEORETICAL RESULTS}

We now return to the main point of this paper, which is the determination of the orientation of a single emitter. We elaborate here on the theory of Sec. II and show that the emitting dipole orientation can be extracted from a polarization analysis.

We distinguish between 1D and 2D dipoles, as the importance of this difference was pointed out in the previous section. The orientation $(\Theta, \Phi)$ of a $1 \mathrm{D}$ dipole is defined as in Sec. I. For a 2D dipole, $(\Theta, \Phi)$ will refer to the orientation of the emitter dark axis [26]: the axis normal to the plane containing the two emitting dipoles. Since the two dipoles are incoherent, the intensity emitted by a $2 \mathrm{D}$ dipole of dark-axis orientation $(\Theta, \Phi)$ can be calculated as a 
sum of the intensities emitted by two 1D dipoles of orientations $(\pi / 2-\Theta, \Phi)$ and $(\pi / 2, \Phi+\pi / 2)$.

After some manipulation, the measured intensity as a function of a rotating polarizer angle $\alpha$ [Eq. (20)] can be written as

$$
\begin{aligned}
& I_{1 D}(\alpha)=I_{\min }+\left(I_{\max }-I_{\min }\right) \cos ^{2}(\Phi-\alpha), \\
& I_{2 D}(\alpha)=I_{\max }+\left(I_{\min }-I_{\max }\right) \cos ^{2}(\Phi-\alpha) .
\end{aligned}
$$

This expression shows that the emitted intensity is partially polarized for both $1 \mathrm{D}$ and $2 \mathrm{D}$ dipoles, with a Malus component in $\cos ^{2}(\Phi-\alpha)$ from which the emitter in-plane orientation $\Phi$ can be straightforwardly extracted. Let us stress, however, that $\Phi$ is obtained as the polarizer angle that maximizes the detected intensity for a $1 \mathrm{D}$ dipole but as the angle that minimizes the intensity for a 2D dipole (because $\Phi$ is the orientation of the "dark axis").

We now express the maximum intensity $I_{\max }$ and minimum intensity $I_{\min }$ and show that their knowledge can lead to $\Theta$. For a 1D dipole,

$$
\begin{gathered}
I_{\min }=A \sin ^{2} \Theta+B \cos ^{2} \Theta, \\
I_{\max }-I_{\min }=C \sin ^{2} \Theta,
\end{gathered}
$$

and for a $2 \mathrm{D}$ dipole,

$$
\begin{gathered}
I_{\min }=A+B+(A-B+C) \cos ^{2} \Theta, \\
I_{\max }-I_{\min }=C \sin ^{2} \Theta,
\end{gathered}
$$

with, for both $1 \mathrm{D}$ and 2D dipoles, the constants

$$
\begin{gathered}
A=\int_{\theta_{1}=0}^{\theta_{1 \max }} D^{2} \frac{\pi}{4} n_{j} \frac{\cos \theta_{2}}{\cos \theta_{1}}\left|f_{s}-\cos \theta_{1} f_{p_{b}}\right|^{2} \sin \theta_{1} d \theta_{1}, \\
B=\int_{\theta_{1}=0}^{\theta_{1 \max }} D^{2} \pi n_{j} \frac{\cos \theta_{2}}{\cos \theta_{1}}\left|f_{p_{a}}\right|^{2} \sin ^{3} \theta_{1} d \theta_{1}, \\
C=\int_{\theta_{1}=0}^{\theta_{1 \max }} D^{2} \frac{\pi}{2} n_{j} \frac{\cos \theta_{2}}{\cos \theta_{1}}\left|\cos \theta_{1} f_{p_{a}}+f_{s}\right|^{2} \sin \theta_{1} d \theta_{1},
\end{gathered}
$$

with $j=1$ for cases (ii) and (iii) and $j=2$ for case (iv).

From an experimental perspective, the measured $I_{\max }$ and $I_{\min }$ are both proportional to the total emitted intensity, and we define the degree of linear polarization of the emission as

$$
\delta(\Theta)=\frac{I_{\max }-I_{\min }}{I_{\max }+I_{\min }} .
$$

Including Eqs. (27) to (30) in Eqs. (25) and (26), one obtains, for a $1 \mathrm{D}$ dipole and a 2D dipole, respectively,

$$
\begin{gathered}
\delta(\Theta)_{1 D}=\frac{C \sin ^{2} \Theta}{(2 A-2 B+C) \sin ^{2} \Theta+2 B}, \\
\delta(\Theta)_{2 D}=\frac{C \sin ^{2} \Theta}{-(2 A-2 B+C) \sin ^{2} \Theta+4 A+2 C} .
\end{gathered}
$$

In the case of a vertical dipole $(\Theta=0)$, the emission is fully unpolarized $(\delta=0)$ for both $1 \mathrm{D}$ and $2 \mathrm{D}$ dipoles, as expected given the cylindrical symmetry of the system. As the angle $\Theta$ is increased, the emission becomes more polarized and, for $\Theta=\pi / 2, \delta$ reaches a maximum value of $C /(2 A+C)$ for a 1D dipole and $C /(2 A+2 B+C)$ for a $2 \mathrm{D}$ dipole, which is always smaller than unity: The emission is never strictly fully polarized.

These equations show that, for both $1 \mathrm{D}$ and 2D dipoles, it is possible to extract the out-of-plane orientation $\Theta$ from the measured degree of polarization $\delta$. This requires the knowledge of the coefficients $A, B$, and $C$, which can be calculated theoretically for a given situation and can depend on the sample configuration (the presence of an interface) through the functions $f_{s}, f_{p 1}$, and $f_{p 2}$, and on the objective numerical aperture through $\theta_{j \max }$.

Let us briefly discuss the difference between excitation and emission polarization analysis. A typical excitation polarization analysis setup will include a rotating polarizer of angle $\alpha_{\mathrm{exc}}$ on the path of the excitation beam, and it will measure the emitted intensity $I\left(\alpha_{\text {exc }}\right)$. It has been shown in Ref. [17] that the orientation of the exciting electric field $\vec{E}_{\text {exc }}$, at the position of the emitter, is very close to the orientation $\vec{u}_{\alpha \text { exc }}$ of the excitation polarizer, even when taking into account emitter-positioning imperfections and a high-objective numerical aperture. For this reason, for a 1D dipole, one can write

$$
\begin{aligned}
I\left(\alpha_{\mathrm{exc}}\right) & \propto\left|\vec{d} \cdot \vec{E}_{\mathrm{exc}}\right|^{2} \\
& \propto I_{0}\left|\vec{u}_{d} \cdot \vec{u}_{\alpha}\right|^{2}=I_{0} \cos ^{2}\left(\Phi-\alpha_{\mathrm{exc}}\right) \sin ^{2} \Theta .
\end{aligned}
$$

In this case, as pointed out in earlier work on singlemolecule orientation $[1,10]$, the in-plane angle $\Phi$ can be obtained as the angle $\alpha_{\text {exc }}$ that maximizes $I\left(\alpha_{\text {exc }}\right)$, but the out-of-plane angle $\Theta$ cannot be obtained because the value $I_{0}$ is not known. It is clear, by comparison of expressions (25) and (37), that the polarization analyses in excitation and emission are two very different situations.

We now discuss the calculated correspondence between $\Theta$ and $\delta$ and analyze its physical meaning.

We start with an emitter in a homogeneous medium [situation (i)]. The values of $A, B$, and $C$ can then be calculated analytically [49], and in the limit of high numerical aperture $\left(\theta_{\max }=\pi / 2\right)$, we find for a 1D dipole the simple expression

$$
\delta_{\text {high NA }}(\Theta)=\frac{7}{8} \sin ^{2} \Theta
$$


so that $\delta$ ranges from 0 to 0.875 . On the other hand, for a $1 \mathrm{D}$ dipole in the limit of a low numerical aperture, a secondorder development in $\theta_{\max }$ leads to

$$
\delta_{\text {low NA }}(\Theta)=\frac{\sin ^{2} \Theta}{\left(1-\left(\left(\theta_{\max }^{2}\right) / 2\right)\right) \sin ^{2} \Theta+\left(\left(\theta_{\max }^{2}\right) / 2\right)} .
$$

In this case, the maximum value of $\delta$ is 1 . The case of $\theta_{\max } \sim 0$ (very low numerical aperture) is interesting, as it corresponds to probing a single direction of emission. Our calculations show that, for $\theta_{\max } \sim 0, \delta$ is unity for any $\Theta$, as expected since the emission of a 1D dipole into a specific direction is always polarized (the case $\Theta=0$ is an exception; it gives $\delta=0$ but is actually not measurable because no light can be detected for this orientation; a 1D dipole never emits into the direction of its axis). This means that a very low numerical aperture is not an appropriate condition for measuring the orientation of an emitter by polarization analysis: The angle $\Theta$ cannot be deduced from the value of $\delta$, as it is always unity. As the numerical aperture is increased, the objective collects the emission into different directions, each direction having a specific polarization; thus, the collected beam is a summation of different polarizations and has a lower degree of polarization. It is this summation that allows the measurement of $\Theta$ from the polarization properties. As for a $2 \mathrm{D}$ dipole in a homogeneous medium, it can also be calculated analytically for the limiting case of high numerical aperture $\left(\theta_{\max }=\pi / 2\right)$ :

$$
\delta_{\text {high NA }}(\Theta)=\frac{7}{16} \sin ^{2} \Theta,
$$

which is half the degree of polarization in the 1D case [similar trends are also observed for situations (ii) and (iv) in Figs. 5(a) and 5(b)]. As expected, a 2D dipole emits with a lower degree of polarization since it corresponds to a sum of two incoherent dipoles. In the limit of low numerical aperture,

$$
\delta_{\text {low NA }}(\Theta)=\frac{\sin ^{2} \Theta}{\left(\left(\left(\theta_{\max }^{2}\right) / 2\right)-1\right) \sin ^{2} \Theta+2} .
$$

For $\theta_{\max } \sim 0$, which corresponds to probing a single direction of emission, $\delta$ is not unity, except for the case $\Theta=90^{\circ}$, unlike the case of a $1 \mathrm{D}$ dipole. This is explained by the fact that, even when probing a single direction, the emission is not necessarily polarized, as it is a sum of the emissions of two incoherent dipoles with different orientations.

We now turn to the cases in which the emitter is near an interface. We have introduced in Sec. I several standard experimental conditions. We plot in Fig. 5 the relation between $\Theta$ and $\delta$ for the following parameters: substrate index $=1.5$, other mediumindex $=1, d=50 \mathrm{~nm}$,
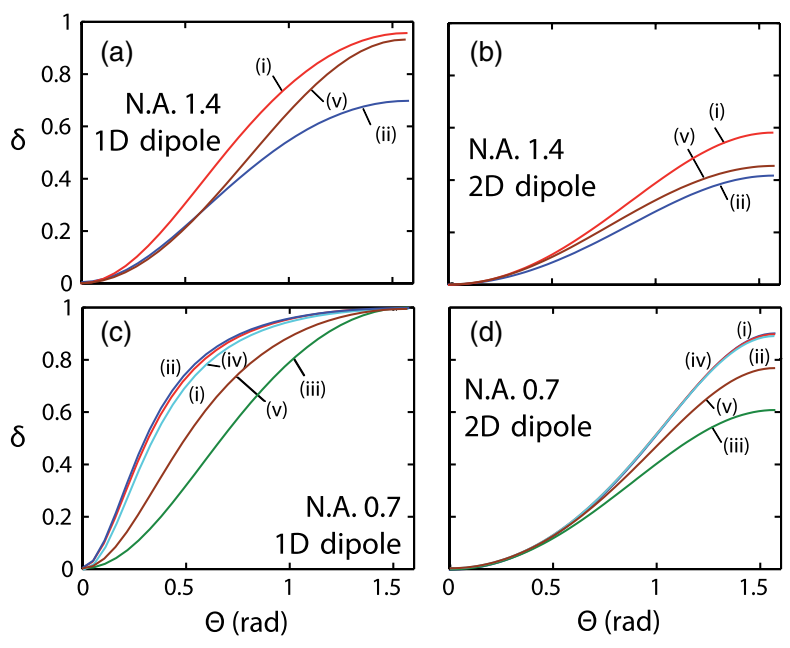

FIG. 5. Theoretical value of $\delta$ as a function of the angle $\Theta$ for a $1 \mathrm{D}$ dipole $(\mathrm{a}, \mathrm{c})$ or a 2D dipole $(\mathrm{b}, \mathrm{d})$ with numerical aperture 1.4 (a,b) or 0.7 (c,d), calculated in situations (i), (ii), and (v) for (a,b) and situations (i)-(v) for (c,d), with $d=50 \mathrm{~nm}$ for cases (ii) and (iv) and $d=0$ for cases (iii) and (v), and $\lambda=620 \mathrm{~nm}$.

$\lambda=620 \mathrm{~nm}$. We consider the case of a 0.7 numerical aperture for situations (i)-(v) and the case of an immersion objective with 1.4 numerical aperture for situations (i), (ii), and (v). We distinguish between 1D and 2D dipoles. The curves obtained show a similar trend, with an increase from $\delta(0)=0$ to a maximum $\delta(\pi / 2)$, which is always below 1 . However, the quantitative differences between these curves are significant. For a given value of $\delta$, depending on the experimental configuration and on the 1D or 2D nature of the dipole, the corresponding values of $\Theta$ can be different by up to $65^{\circ}$. It is thus possible, in all these experimental configurations, to extract $\Theta$ from $\delta$, but only if the specificities of this configuration (numerical aperture, $1 \mathrm{D} / 2 \mathrm{D}$ nature, index, interface, etc.) are properly included in the theoretical analysis.

In Fig. 5, we note that the $\Theta$ dependence of $\delta$ is similar for all cases. This remark suggests that, for close experimental conditions (small distance $d$ between the emitter and the interface), the expected behavior $\delta=f(\theta)$ is the same whether the emitter is slightly above or below the surface.

Finally, let us discuss the polarization analysis in the case of nanorod emission [50-53]. In these studies, the nanorods are assumed to be horizontally deposited $(\Theta=\pi / 2)$, and the degree of linear polarization $\delta$ is measured in order to probe to what extent the rod behaves as a linear dipole. Our curves show that, if the rod is a perfect 1D dipole, a value $\delta \simeq 1$ should be measured in all situations [case (v) excepted] for a 0.7 numerical aperture, but, for a 1.4 numerical aperture, values of $\delta$ between 0.7 and 0.98 are calculated, depending on the situation. This must be taken into account when interpreting the experimental values of $\delta$, which range from 0.7 to 0.9 [51-53]. 


\section{EXPERIMENTAL ORIENTATION MEASUREMENT}

In this section, we apply these considerations to demonstrate experimental orientation measurements on $\mathrm{CdSe} / \mathrm{CdS}$ nanocrystals, chosen for their brightness and photostability.

We use an inverted microscope to study a sample of $\mathrm{CdSe} / \mathrm{CdS}$ nanocrystals on a glass substrate, covered by $50 \mathrm{~nm}$ of polymethyl methacrylate (PMMA) (measured by a profilometer), with an oil-immersion objective (1.4 numerical aperture, x100). A single nanocrystal is excited by a diode laser at $450 \mathrm{~nm}$ (for each measurement, a standard Hanbury-Brown and Twiss measurement demonstrates the emission of a single photon and, therefore, the imaging of a single emitter [54]). The photoluminescence is collected by the same objective and focused on a $100-\mu \mathrm{m}$ pinhole in order to spatially filter the background noise. It is then recollimated, passed through a half-wave plate, and separated into two arms by a polarizing beam-splitter cube; each arm is focused on a single-photon counting avalanche photodiode (see inset of Fig. 6). The half-wave plate is continuously rotated with an angle $\alpha / 2$, and the photoluminescence intensity is measured on each photodiode. The role of the half-wave plate and polarizing cube is equivalent to a polarizer of angle $\alpha$.

We plot in Fig. 6 the intensity on one photodiode, normalized by the sum of the intensities on two photodiodes in order to cancel the fluctuations of the total emitted intensity due to slight emitter instabilities. This curve is well fitted by Eq. (26), with three fitting parameters: $I_{\max }$,

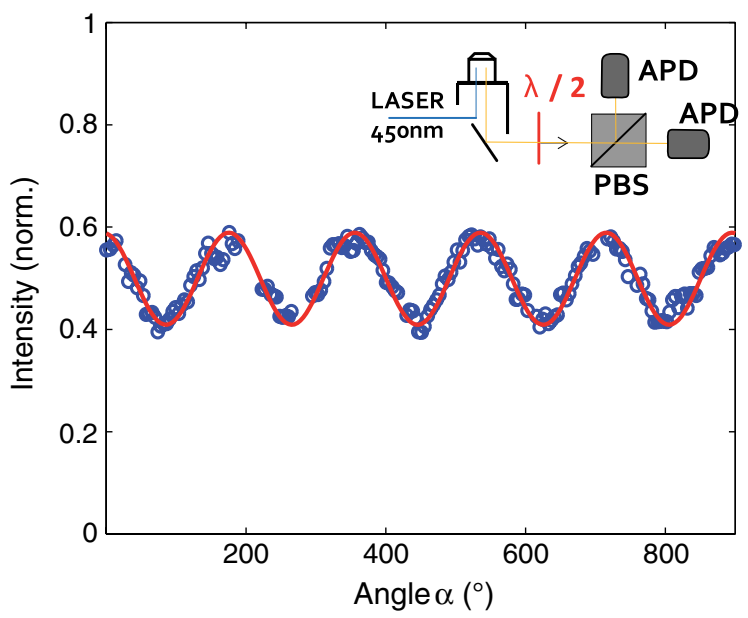

FIG. 6. Circles: Dependence of detected intensity as a function of the polarization analysis angle for a nanocrystal of $\mathrm{CdSe} / \mathrm{CdS}$ in the vicinity of an air-dielectric interface. This curve is normalized by the total intensity detected on both photodiodes in order to account for fluctuations of the total emitted intensity. The different detection efficiencies of the two paths are corrected so that the normalized curve has a mean value of 0.5 . The fitted curve (solid red line) corresponds to Eq. (26). From the fit, we deduce that $\Theta=44^{\circ}$ and $\Phi=52^{\circ}$.
$I_{\min }-I_{\max }$, and $\Phi$. We find, for this nanocrystal, an in-plane angle $\Phi=50^{\circ}$ and a degree of polarization $\delta=$ $18 \%$ from which we deduce, given the theoretical curve of Fig. 5(b) [situation (ii)], an out-of-plane angle $\Theta=43^{\circ}$. We estimate the precision of our fit to $\pm 4^{\circ}$ for $\Phi$ and $\pm 2^{\circ}$ on $\Theta$.

We repeat this measurement for a collection of the $\mathrm{CdSe} /$ $\mathrm{CdS}$ nanocrystals and plot in Fig. 7(a) a histogram of the obtained values of $\delta$. We find values of $\delta$ below 0.4 , in agreement with our theoretical calculation [Fig. 5(b), situation (ii)] that the value of $\delta$ is between 0 and 0.4 for a $2 \mathrm{D}$ dipole in this configuration. This is consistent with our previous demonstration that the $\mathrm{CdSe} / \mathrm{CdS}$ nanocrystals are $2 \mathrm{D}$ emitters. On the other hand, we perform the same measurements for a collection of $\mathrm{CdSe} / \mathrm{ZnS}$ nanocrystals [Fig. 7(a)], and we find values of $\delta$ up to 0.7. These values are too high for a 2D dipole, as shown by Fig. 5(b) (for the $\mathrm{CdSe} / \mathrm{ZnS}$ nanocrystals of emission wavelength $\lambda=565 \mathrm{~nm}$, the calculated curve is not shown here but very close to the case $\lambda=620 \mathrm{~nm}$ ). They could be explained by a mixture of $1 \mathrm{D}$ and $2 \mathrm{D}$ dipoles, as already proposed in Sec. II, since the maximum theoretical $\delta$ is 0.7 for a 1D dipole.

We plot in Fig. 7(b) a histogram of the out-of-plane angles $\Theta$ measured for the $\mathrm{CdSe} / \mathrm{CdS}$ nanocrystals. We find a distribution of angles between $31^{\circ}$ and $83^{\circ}$. We did not find any nanocrystal with orientation $\Theta$ below $30^{\circ}$. Indeed, small values of $\Theta$ are theoretically less likely: For an isotropic distribution of orientations $(\Theta, \Phi)$, only $13 \%$ of orientations show $\Theta<30^{\circ}$. It is also possible that the
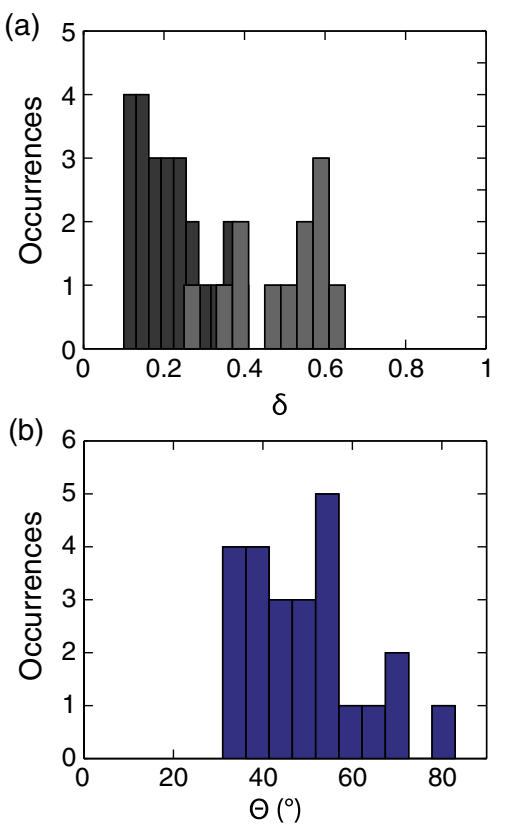

FIG. 7. (a) Histogram of experimental values of $\delta$ for $24 \mathrm{CdSe} /$ $\mathrm{CdS}$ nanocrystals (black bars) and $13 \mathrm{CdSe} / \mathrm{ZnS}$ nanocrystals (grey bars). (b) Histogram of experimental values of $\Theta$ for 24 $\mathrm{CdSe} / \mathrm{CdS}$ nanocrystals. 
predominance of the $40^{\circ}-60^{\circ}$ orientations is due to the specific geometry of the $\mathrm{CdSe} / \mathrm{CdS}$ nanocrystals. These thick-shell NCs are not spherical, and they display a faceted geometry [Fig. 4(d)]. They tend to have a bipyramidal shape, and they may lie on the substrate with some preferred orientation, which could explain the trend we observed in the polarization measurements.

\section{VICINITY OF A GOLD SURFACE}

Finally, we discuss in this last section the orientation measurement for an emitter in the vicinity of a gold film. This situation is of interest, for instance, in the context of coupling to surface plasmons [11] or nanoantennas [13], for which the orientation is crucial. On a glass substrate, we deposit $200 \mathrm{~nm}$ of gold, $25 \mathrm{~nm}$ of silica, CdSe/CdS nanocrystals, and $50 \mathrm{~nm}$ of PMMA [Fig. 8(a)]. Fluorescence is collected by an oil-immersion objective of numerical aperture 1.4.

Since the optical skin depth in gold is a few tens of nanometres, the 200-nm gold layer can be considered infinitely thick. This is situation (ii), where $n_{1}=1.5$ is the silica/PMMA index, $d=25 \mathrm{~nm}$ is the distance to the gold film, $n_{2}$ is the gold dielectric constant (obtained from ellipsometric measurements), and $\lambda=620 \mathrm{~nm}$. We plot in Fig. 8(b) the emission pattern, for three different angles $\Theta$. For clarity, we plot it in the 2D angular coordinates $\theta_{1}$ and $\phi$, expressed in Cartesian coordinates $\left(\tan \theta_{1} \times \cos \phi\right)$ and $\left(\tan \theta_{1} \times \sin \phi\right)$. Apart from the total emitted intensity (which is not a useful quantity to deduce $\Theta$, as the emitted intensity can vary significantly among nanocrystals), there are very little differences between these images, so $\Theta$ cannot be obtained. Moreover, the emission patterns have rotation invariance, so $\Phi$ cannot be known from these patterns either. This is an example of a situation for which defocused imaging, which probes the emission pattern of a dipole, cannot be used to measure the orientation of a dipole.

Polarization analysis, on the other hand, is appropriate in this configuration. We plot in Fig. 8(c) the theoretical dependence of $\delta$ on $\Theta$. This curve shows a sufficiently clear dependence for $\Theta$ to be determined if $\delta$ is known. We plot in Fig. 8(d) the experimental polarization analysis for a $\mathrm{CdSe} / \mathrm{CdS}$ nanocrystal. By fitting these data with Eq. (26), we find the nanocrystal orientation: $\Phi=69 \pm 3^{\circ}$ and $\Theta=46 \pm 1^{\circ}$.

We make the same measurements for 12 nanocrystals on this gold-silica substrate and plot a histogram of the measured values of $\delta$ in Fig. 8(e) and of the corresponding $\Theta$ in Fig. 8(f). The values of $\Theta$ are distributed between 0.1 and 0.4 , in agreement with the calculations of Fig. 8(c), showing that $\delta$ can be between 0 and 0.7 . The corresponding orientations $\Theta$ are between 25 and $55^{\circ}$. These angles are consistent with the values obtained in Sec. IV. (a)
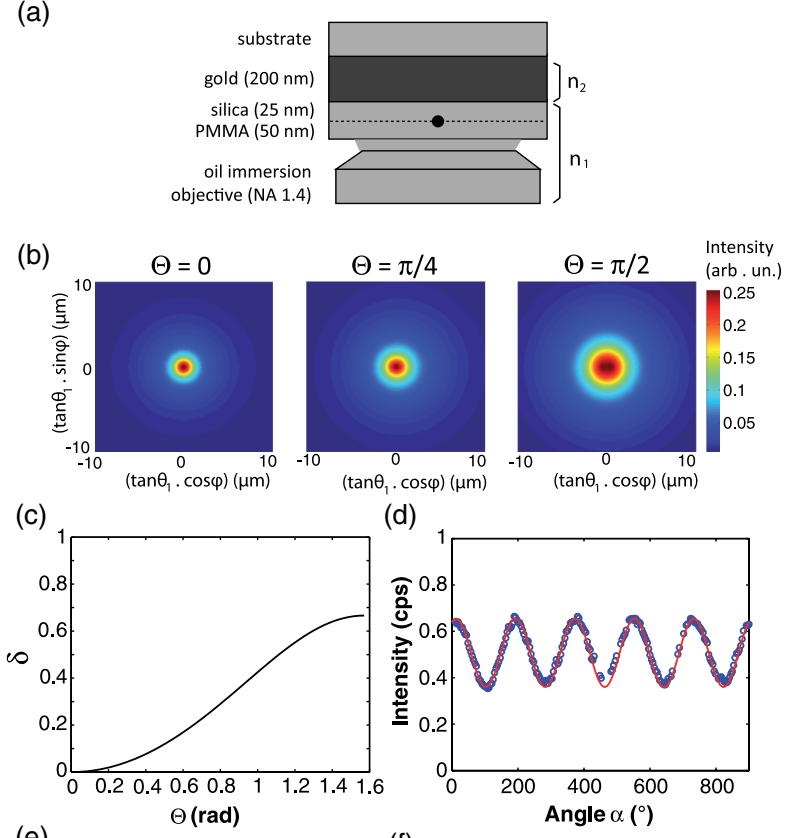

(d)
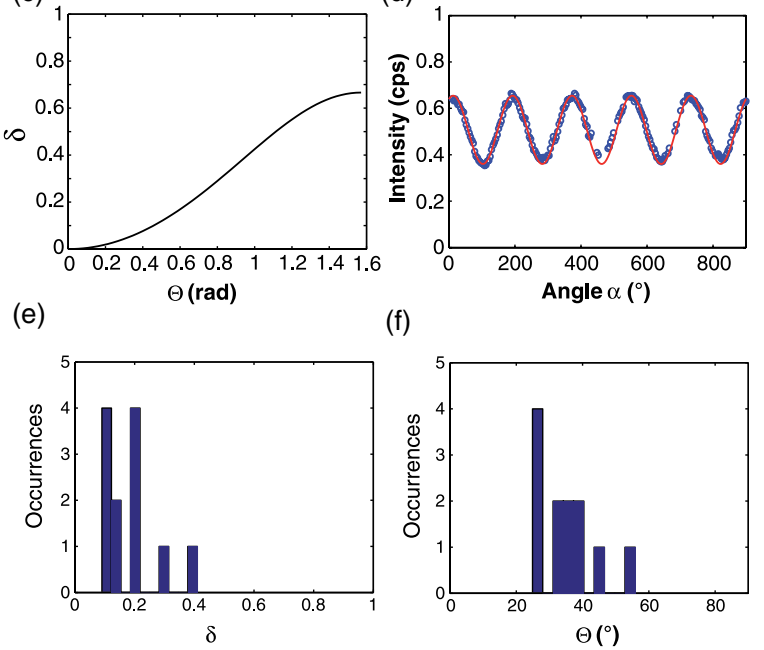

(f)

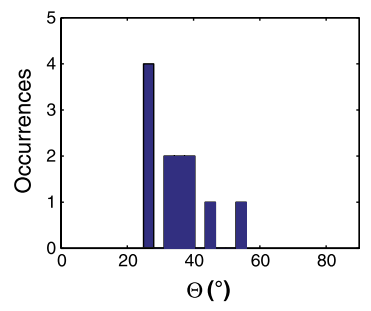

FIG. 8. (a) Schematic of the experimental system. (b) Calculated emission pattern for a $2 \mathrm{D}$ dipole with $(\Theta=0, \Phi=0)$, $(\Theta=(\pi / 4), \Phi=0)$, and $(\Theta=(\pi / 2), \Phi=0)$. (c) Calculated values of $\delta$ as a function of the angle $\Theta$ for a $2 \mathrm{D}$ dipole ( $c$-axis inclination). (d) Circles: Dependence of the $x$-polarized emission intensity as a function of the half-wave plate angle for a nanocrystal of $\mathrm{CdSe} / \mathrm{CdS}$ in the vicinity of gold-dielectric interface, fitted (solid red line) with Eq. (26). From the fit, we deduce that $\Theta=46^{\circ}$ and $\Phi=69^{\circ}$. (e) and (f) Histograms of experimental values of $\delta$ and $\Theta$ for $12 \mathrm{CdSe} / \mathrm{CdS}$ nanocrystals situated $50 \mathrm{~nm}$ from a gold surface.

\section{CONCLUSION}

In this paper, we addressed the orientation measurement of a single photoluminescent emitter. We showed that, in contrast with excitation polarization analysis, emission polarization analysis provides both the in-plane angle $\Phi$ and the out-of-plane angle $\Theta$. We developed a model of the polarization analysis experiment and insisted on the importance of taking into account the sample geometry (the presence of an interface) and the objective numerical aperture. We distinguished five different sample configurations, which we believe will cover most experimental conditions. We showed that the angle $\Theta$ can be deduced from the measured degree of polarization $\delta$, which is established analytically. These expressions can lead to 
rather different quantitative values, depending on the experimental situation and on the nature of the dipole, which have to be carefully taken into account in the model. We applied this method to demonstrate experimentally the orientation of $\mathrm{CdSe} / \mathrm{CdS}$ nanocrystals, which we proved to be $2 \mathrm{D}$ dipoles. In the case encountered in plasmonics of a 2D dipole near a gold film, the wellestablished defocused imaging cannot yield precise results on its orientation, though we proved that polarization analysis is an efficient method.

Deterministic coupling of nanoemitters to photonic nanostructures is a major issue for optimizing light-matter interaction. After years of development, the deterministic positioning of nanoemitters right at the maximum of cavity fields is starting to be well controlled. However, deterministic orientation of dipoles, which is as crucial as spatial positioning for coupling emission to cavity modes, remains a challenge. The polarimetric method proposed in this paper is about to be implemented in a lithographic process for selecting well-oriented dipoles. It opens the way to the deterministic control of dipole orientation inside photonic and especially plasmonic systems.

\section{ACKNOWLEDGMENTS}

The authors would like to thank Francis Breton, Stéphane Chenot, and Loïc Becerra for technical support and fabrication of the samples, as well as Jean-Louis Fave, Michel Schott, and Paul Bénalloul for fruitful discussions. This work was funded by the Agence Nationale de la Recherche (P3N Delight) and by the Centre de Compétence NanoSciences Ile-de-France (C'Nano IdF).

\section{APPENDIX}

In case (v), one must add to the equations of case (iv) the evanescent component of the dipole's emission to the farfield component [Eq. (1)]. This new component involves the $\vec{k}_{1}$ vectors that fulfill the condition $k_{1} \leq k_{\|_{1}} \leq k_{2}$, where $k_{1}$ and $k_{2}$ stand for the wave vectors in media 1 and 2, respectively. In this particular case, both Eqs. (1) and (4) must be modified, in order to describe the added component, by replacing the unit vectors $\vec{u}_{k}$ by $\vec{u}_{k}^{*}$ and $\vec{u}_{p}$ by $\vec{u}_{p}^{*}$, which is defined as

$$
\vec{u}_{k}^{*}=\frac{1}{k_{1}}\left(\begin{array}{c}
k_{\|_{1}} \cos \phi \\
k_{\|_{1}} \sin \phi \\
i \kappa
\end{array}\right)=\left(\begin{array}{c}
\cosh \beta \cos \phi \\
\cosh \beta \sin \phi \\
i \sinh \beta
\end{array}\right)
$$

and

$$
\vec{u}_{p}^{*}=\left(\begin{array}{c}
i \sinh \beta \cos \phi \\
i \sinh \beta \sin \phi \\
-\cosh \beta
\end{array}\right)
$$

with $\kappa$ the real part of the $z$ component of the wave vector $\vec{k}_{1}$ and $\beta$ the angle, which verify

$$
k_{\|_{1}}^{2}-\kappa^{2}=k_{1}^{2}
$$

and

$$
\cosh \beta=\frac{k_{\|_{1}}}{k_{1}} \quad \text { and } \quad \sinh \beta=\frac{\kappa}{k_{1}} .
$$

In the same way, in order to verify Eq. (4), $E_{p_{a}}\left(\theta_{1}, \phi\right)$ and $E_{p_{b}}\left(\theta_{1}, \phi\right)$, defined in Eqs. (8) and (9), have to be changed into

$$
E_{p_{a}}^{*}(\beta, \phi)=-\cos \Theta \cosh \beta
$$

and

$$
E_{p_{b}}^{*}(\beta, \phi)=i \sin \Theta \sinh \beta \cos (\Phi-\phi) .
$$

The $s$ component of the electric field is expressed as

$$
E_{s}^{*}(\beta, \phi)=\sin \Theta \sin (\phi-\Phi),
$$

just as in Eq. (6).

One must introduce, as above, the functions $f_{s}^{*}, f_{p_{a}}^{*}$, and $f_{p_{b}}^{*}$, whose definitions for case (v) are

\begin{tabular}{cccc}
\hline & $f_{s}^{*}=$ & $f_{p_{a}}^{*}=$ & $f_{p_{b}}^{*}=$ \\
\hline (v) & $t_{s}^{* 12} e^{-\kappa d}$ & $t_{p}^{* 12} e^{-\kappa d}$ & $t_{p}^{* 12} e^{-\kappa d}$ \\
\hline
\end{tabular}

with

$$
\begin{aligned}
& t_{s}^{* 12}=\frac{2 i n_{1} \sinh \beta}{n_{2} \cos \theta_{2}+i n_{1} \sinh \beta}, \\
& t_{p}^{* 12}=\frac{2 i n_{1} \sinh \beta}{n_{1} \cos \theta_{2}+i n_{2} \sinh \beta},
\end{aligned}
$$

with

$$
n_{1} \cosh \beta=n_{2} \sin \theta_{2} .
$$

By taking into account the apodization factor $\left|\cos \theta_{2} / \sinh \beta\right|^{2}$ for the transmission case (v), the evanescent component of the emitted power is finally expressed as

$$
\begin{aligned}
P^{*}(\alpha)= & \int_{\varphi=0}^{2 \pi} \int_{\theta_{2}=\operatorname{arc} \sin \frac{n_{1}}{n_{2}}}^{\theta_{2 \max }}\left|\overrightarrow{\mathcal{E}}^{*}\left(\theta_{1}, \varphi\right) \cdot \vec{u}(\alpha)\right|^{2} \\
& \times\left|\frac{\cos \theta_{2}}{\sinh \beta}\right|^{2} n_{2} \sin \theta_{2} d \theta_{2} d \varphi,
\end{aligned}
$$

with 


$$
\begin{aligned}
\overrightarrow{\mathcal{E}}^{*}(\beta, \phi)= & D \frac{n_{2}}{n_{1}}\left(E_{s}^{*}(\beta, \phi) f_{s}^{*}(\beta) \vec{v}_{s}\right. \\
& \left.+\left[E_{p_{a}}^{*}(\beta, \phi) f_{p_{a}}^{*}(\beta)+E_{p_{b}}^{*}(\beta, \phi) f_{p_{b}}^{*}(\beta)\right] \vec{v}_{p}\right) .
\end{aligned}
$$

Finally, for the particular case (v), the total detected power is then $P^{*}(\alpha)$ added to $P^{(i v)}(\alpha)$, the power calculated for case (iv) in Eq. (21):

$$
P^{(v)}(\alpha)=P^{(i v)}(\alpha)+P^{*}(\alpha) .
$$

In this case, Eqs. (31), (32), and (33) must be modified to the following:

$$
\begin{aligned}
& A^{(v)}=A+A^{*}, \\
& B^{(v)}=B+B^{*}, \\
& C^{(v)}=C+C^{*},
\end{aligned}
$$

where

$$
\begin{aligned}
& A^{*}= \int_{\theta_{2}=\operatorname{arc~sin} \frac{n_{1}}{n_{2}}}^{\theta_{2 \max }} D^{2} \frac{\pi}{4}\left|\frac{\cos \theta_{2}}{\sinh \beta}\right|^{2} \\
& \times n_{2}\left(\frac{n_{2}}{n_{1}}\right)^{2}\left|f_{s}^{*}-i \sinh \beta f_{p_{b}}^{*}\right|^{2} \sin \theta_{2} d \theta_{2}, \\
& B^{*}=\int_{\theta_{2}=\arcsin \frac{n_{n}}{n_{2}}}^{\theta_{2 \max }} D^{2} \pi\left|\frac{\cos \theta_{2}}{\sinh \beta}\right|^{2} n_{2}\left(\frac{n_{2}}{n_{1}}\right)^{2}\left|f_{p_{a}}^{*} \cosh \beta\right|^{2} \sin \theta_{2} d \theta_{2}, \\
& C^{*}=\int_{\theta_{2}=\operatorname{arc~sin} \frac{n_{1}}{n_{2}}}^{\theta_{2 \max }} D^{2} \frac{\pi}{2}\left|\frac{\cos \theta_{2}}{\sinh \beta}\right|^{2} \\
& \times n_{2}\left(\frac{n_{2}}{n_{1}}\right)^{2}\left|i \sinh \beta f_{p_{b}}^{*}+f_{s}^{*}\right|^{2} \sin \theta_{2} d \theta_{2} .
\end{aligned}
$$

[1] T. Ha, Th. Enderle, D. S. Chemla, P. R. Selvin, and S. Weiss, Single Molecule Dynamics Studied by Polarization Modulation, Phys. Rev. Lett. 77, 3979 (1996).

[2] S. Nie, D. T. Chiu, and R. N. Zare, Probing Individual Molecules with Confocal Fluorescence Microscopy, Science 266, 1018 (1994).

[3] J. K. Trautman and J. J. Macklin, Time-Resolved Spectroscopy of Single Molecules Using Near-Field and Far-Field Optics, Chem. Phys. 205, 221 (1996).

[4] E. Betzig and J. Chichester, Single Molecules Observed by Near-Field Scanning Optical Microscopy, Science 262, 1422 (1993).

[5] M. Orrit and J. Bernard, Single Pentacene Molecules Detected by Fluorescence Excitation in a p-Terphenyl Crystal, Phys. Rev. Lett. 65, 2716 (1990).
[6] W. Trabesinger, A. Renn, B. Hecht, U. P. Wild, A. Montali, P. Smith, and C. Weder, Single-Molecule Imaging Revealing the Deformation-Induced Formation of a Molecular Polymer Blend, J. Phys. Chem. B 104, 5221 (2000).

[7] J. N. Forkey, M. E. Quinlan, M. A. Shaw, J. E. T. Corrie, and Y. E. Goldman, Three-Dimensional Structural Dynamics of Myosin V by Single-Molecule Fluorescence Polarization, Nature (London) 422, 399 (2003).

[8] E. Toprak, J. Enderlein, S. Syed, S. A. McKinney, R. G. Petschek, T. Ha, Y.E. Goldman, and P. R. Selvin, Defocused Orientation and Position Imaging (DOPI) of Myosin V, Proc. Natl. Acad. Sci. U.S.A. 103, 6495 (2006).

[9] X. Brokmann, M.-V. Ehrensperger, J.-P. Hermier, A. Triller, and M. Dahan, Orientational Imaging and Tracking of Single CdSe Nanocrystals by Defocused Microscopy, Chem. Phys. Lett. 406, 210 (2005).

[10] J. J. Macklin, J. K. Trautman, T. D. Harris, and L. E. Brus, Imaging and Time-Resolved Spectroscopy of Single Molecules at an Interface, Science 272, 255 (1996).

[11] C. Vion, P. Spinicelli, L. Coolen, C. Barthou, J.-M. Frigerio, J.-P. Hermier, and A. Maître, Controlled Modification of Single Colloidal CdSe/ZnS Quantum Dots Fluorescence through Interactions with Gold Surface Plasmons, Opt. Express 18, 7440 (2010).

[12] P. Bharadwaj, B. Deutsch, and L. Novotny, Optical Antennas, Adv. Opt. Photonics 1, 438 (2009).

[13] C. Belacel, B. Habert, F. Bigourdan, F. Marquier, J.-P. Hugonin, S. Michaelis de Vasconcellos, X. Lafosse, L. Coolen, C. Schwob, C. Javaux, B. Dubertret, J.-J. Greffet, P. Senellart, and A. Maître, Controlling Spontaneous Emission with Plasmonic Optical Patch Antennas, Nano Lett. 13, 1516 (2013).

[14] A. Badolato, K. Hennessy, M. Atatüre, J. Dreiser, E. Hu, P. M. Petroff, and A. Imamoglu, Deterministic Coupling of Single Quantum Dots to Single Nanocavity Modes, Science 308, 1158 (2005).

[15] O. Gazzano, S. Michaelis de Vasconcellos, K. Gauthron, C. Symonds, J. Bloch, P. Voisin1, J. Bellessa, A. Lemaître, and P. Senellart, Evidence for Confined Tamm Plasmon Modes under Metallic Microdisks and Application to the Control of Spontaneous Optical Emission, Phys. Rev. Lett. 107, 247402 (2011).

[16] K. Hennessy, C. Högerle, E. Hu, A. Badolato, and A. Imamoğlu, Tuning Photonic Nanocavities by Atomic Force Microscope Nano-oxidation, Appl. Phys. Lett. 89, 041118 (2006).

[17] T. Ha, T. A. Laurence, D. S. Chemla, and S. Weiss, Polarization Spectroscopy of Single Fluorescent Molecules, J. Phys. Chem. B 103, 6839 (1999).

[18] F. Güttler, M. Croci, A. Renn, and U. P. Wild, Single Molecule Polarization Spectroscopy: Pentacene in p-Terphenyl, Chem. Phys. 211, 421 (1996).

[19] M. A. Lieb and A. J. Meixner, A High Numerical Aperture Parabolic Mirror as Imaging Device for Confocal Microscopy, Opt. Express 8, 458 (2001).

[20] L. Novotny, M. R. Beversluis, K. S. Youngworth, and T. G. Brown, Longitudinal Field Modes Probed by Single Molecules, Phys. Rev. Lett. 86, 5251 (2001).

[21] B. Sick, B. Hecht, and L. Novotny, Orientational Imaging of Single Molecules by Annular Illumination, Phys. Rev. Lett. 85, 4482 (2000). 
[22] R. M. Dickson, D. J. Norris, and W. E. Moerner, Simultaneous Imaging of Individual Molecules Aligned Both Parallel and Perpendicular to the Optic Axis, Phys. Rev. Lett. 81, 5322 (1998).

[23] H. Ishitobi, I. Nakamura, Z. Sekkat, and S. Kawata, Orientational Imaging of Single Molecules by Using Azimuthal and Radial Polarizations, J. Phys. Chem. B 114, 2565 (2010).

[24] R. Pariser, Theory of the Electronic Spectra and Structure of the Polyacenes and of Alternant Hydrocarbons, J. Chem. Phys. 24, 250 (1956).

[25] A. I. Chizhik, A. M. Chizhik, D. Khoptyar, S. Bär, and A. J. Meixner, Excitation Isotropy of Single CdSe/ZnS Nanocrystals, Nano Lett. 11, 1131 (2011).

[26] S. A. Empedocles, R. Neuhauser, and M. G. Bawendi, Three-Dimensional Orientation Measurements of Symmetric Single Chromophores Using Polarization Microscopy, Nature (London) 399, 126 (1999).

[27] I. Chung, K. T. Shimizu, and M. G. Bawendi, Room Temperature Measurements of the 3D Orientation of Single CdSe Quantum Dots Using Polarization Microscopy, Proc. Natl. Acad. Sci. U.S.A. 100, 405 (2003).

[28] X. Brokmann, L. Coolen, M. Dahan, and J.-P. Hermier, Measurement of the Radiative and Nonradiative Decay Rates of Single CdSe Nanocrystals through a Controlled Modification of their Spontaneous Emission, Phys. Rev. Lett. 93, 107403 (2004).

[29] J. Jasny and J. Sepiol, Single Molecules Observed by Immersion Mirror Objective. A Novel Method of Finding the Orientation of a Radiating Dipole, Chem. Phys. Lett. 273, 439 (1997).

[30] A. P. Bartko and R. M. Dickson, Imaging ThreeDimensional Single Molecule Orientations, J. Phys. Chem. B 103, 11237 (1999).

[31] M. Böhmer and J. Enderlein, Orientation Imaging of Single Molecules by Wide-Field Epifluorescence Microscopy, J. Opt. Soc. Am. B 20, 554 (2003).

[32] J. Sepiol, J. Jasny, J. Keller, and U. P. Wild, Single Molecules Observed by Immersion Mirror Objective. The Orientation of Terrylene Molecules via the Direction of its Transition Dipole Moment, Chem. Phys. Lett. 273, 444 (1997).

[33] D. Patra, I. Gregor, and J. Enderlein, Image Analysis of Defocused Single-Molecule Images for Three-Dimensional Molecule Orientation Studies, J. Phys. Chem. A 108, 6836 (2004).

[34] M. A. Lieb, J. Zavislan, and L. Novotny, Single-Molecule Orientations Determined by Direct Emission Pattern Imaging, J. Opt. Soc. Am. B 21, 1210 (2004).

[35] F. Aguet, S. Geissbühler, I. Märki, T. Lasser, and M. Unser, Super-Resolution Orientation Estimation and Localization of Fluorescent Dipoles Using 3-D Steerable Filters, Opt. Express 17, 6829 (2009).

[36] M. Backlund, M.D. Lew, A. S. Backer, S. J. Sahl, G. Grover, A. Agrawal, R. Piestun, and W. E. Moerner, Simultaneous, Accurate Measurement of the 3D Position and Orientation of Single Molecules, Proc. Natl. Acad. Sci. U.S.A. 109, 19087 (2012).

[37] A. P. Bartko and R. M. Dickson, Three-Dimensional Orientations of Polymer-Bound Single Molecules, J. Phys. Chem. B 103, 3053 (1999).

[38] J. Hohlbein and C. G. Hübner, Three-Dimensional Orientation Determination of the Emission Dipoles of Single
Molecules: The Shot-Noise Limit, J. Chem. Phys. 129, 094703 (2008).

[39] J. T. Fourkas, Rapid Determination of the Three-Dimensional Orientation of Single Molecules, Opt. Lett. 26, 211 (2001).

[40] M. Ohmachi, Y. Komori, A. H. Iwane, F. Fujii, T. Jin, and T. Yanagida, Fluorescence Microscopy for Simultaneous Observation of 3D Orientation and Movement and its Application to Quantum Rod-Tagged Myosin V, Proc. Natl. Acad. Sci. U.S.A. 109, 5294 (2012).

[41] W. Lukosz, Light Emission by Multipole Sources in Thin Layers. I. Radiation Patterns of Electric and Magnetic Dipoles, J. Opt. Soc. Am. 71, 744 (1981).

[42] L. Novotny and B. Hecht, Principles of Nano-Optics (Cambridge University Press, Cambridge, England, 2012), 2nd ed.

[43] E. Hecht, Optics, International Edition (Addison Wesley, San Francisco, USA, 2002), 4th ed.

[44] R. J. Epstein, F. M. Mendoza, Y. K. Kato, and D. D. Awschalom, Anisotropic Interactions of a Single Spin and Dark-Spin Spectroscopy in Diamond, Nat. Phys. 1,94 (2005).

[45] Al. L. Efros, M. Rosen, M. Kuno, M. Nirmal, D. J. Norris, and M. G. Bawendi, Band-Edge Exciton in Quantum Dots of Semiconductors with a Degenerate Valence Band: Dark and Bright Exciton States, Phys. Rev. B 54, 4843 (1996).

[46] B. Mahler, P. Spinicelli, S. Buil, X. Quelin, J.-P. Hermier, and B. Dubertret, Towards Non-blinking Colloidal Quantum Dots, Nat. Mater. 7, 659 (2008).

[47] A. Cyphersmith, K. Early, A. Maksov, J. Graham, Y. Wang, and M. Barnes, Disentangling the Role of Linear Transition Dipole in Band-Edge Emission from Single CdSe/ZnS Quantum Dots: Combined Linear Anisotropy and Defocused Emission Pattern Imaging, Appl. Phys. Lett. 97, 121915 (2010).

[48] K. T. Early, K. D. McCarthy, M. Y. Odoi, P. K. Sudeep, T. Emrick, and M. D. Barnes, Linear Dipole Behavior in Single CdSe-Oligo(Phenylene Vinylene) Nanostructures, ACS Nano 3, 453 (2009).

[49] D. Axelrod, Carbocyanine Dye Orientation in Red Cell Membrane Studied by Microscopic Fluorescence Polarization, Biophys. J. 26, 557 (1979).

[50] E. Rothenberg, Y. Ebenstein, M. Kazes, and U. Banin, TwoPhoton Fluorescence Microscopy of Single Semiconductor Quantum Rods: Direct Observation of Highly Polarized Nonlinear Absorption Dipole, J. Phys. Chem. B 108, 2797 (2004).

[51] X. Chen, A. Nazzal, D. Goorskey, M. Xiao, Z. A. Peng, and X. Peng, Polarization Spectroscopy of Single CdSe Quantum Rods, Phys. Rev. B 64, 245304 (2001).

[52] J. Hu, L. Li, W. Yang, L. Manna, L. Wang, and A. P. Alivisatos, Linearly Polarized Emission from Colloidal Semiconductor Quantum Rods, Science 292, 2060 (2001).

[53] F. Pisanello, L. Martiradonna, G. Lemenager, P. Spinicelli, A. Fiore, L. Manna, J.-P. Hermier, R. Cingolani, E. Giacobino, M. De Vittorio, and A. Bramati, Room TemperatureDipolelike Single Photon Source with a Colloidal Dot-inRod, Appl. Phys. Lett. 96, 033101 (2010).

[54] X. Brokmann, E. Giacobino, M. Dahan, and J. P. Hermier, Highly Efficient Triggered Emission of Single Photons by Colloidal CdSe/ZnS Nanocrystals, Appl. Phys. Lett. 85, 712 (2004). 